\title{
A decade of change in diabetes services in a deprived multiethnic inner city area: diabetes in Tower Hamlets
}

\author{
TAHSEEN A CHOWDHURY, BOBBY HUDA
}

\begin{abstract}
Introduction: Diabetes care in deprived multiethnic inner city areas can be challenging. Old models of care need to evolve to take into account demographic change, increasing prevalence of the condition and scarcity of resources.

Strategies for change: A managed clinical network was tasked with implementing the standards of the Diabetes National Service Framework. Working with patients and other stakeholders, the group developed a model of care based around enhanced diabetes care in primary care, with specialist care reserved for more complex patients. Specialist support for primary care clinicians was provided by community-based multidisciplinary team meetings held within each primary care locality (network). Structured programmes for education of patients and health professionals were provided by the specialist team. Subsequently, use of National Diabetes Inpatient Audit (NaDIA) data enabled us to attract additional resources to improve diabetes inpatient care.

Outcomes: Between 2008/9 and 2012/3, completed care plans rose from $10 \%$ to $88 \%$ and attendances for retinal screening rose from $\mathbf{7 2} \%$ to $82.8 \%$. The proportion of patients achieving a combination of blood pressure $\leq 140 / 80 \mathrm{mmHg}$ and cholesterol $\leq 4 \mathrm{mmol} / \mathrm{L}$ rose from $35.3 \%$ to $46.1 \%$, and mean glycated haemoglobin dropped from $62 \mathrm{mmol} / \mathrm{mol}$ to $60 \mathrm{mmol} / \mathrm{mol}(7.80 \%$ to $7.66 \%)$. Referrals to specialist care fell from 25 per week to six per week. NaDIA data also showed significant improvements in inpatient diabetes outcomes.

Conclusions: Partnership between patients, specialists and primary care to improve diabetes care in multiethnic deprived inner city areas can achieve significant improvements in outcomes. Fundamental to the success of this approach is to hold regular clinical meetings between spe-
\end{abstract}

Department of Diabetes and Metabolism, The Royal London Hospital, London, UK

Address for correspondence: $\mathrm{Dr}$ Tahseen A Chowdhury Consultant in Diabetes, 7th Floor, John Harrison House, The Royal London Hospital, London E1 1BB, UK.

Tel: +44 (0)20 82238384

E-mail: Tahseen.Chowdhury@bartshealth.nhs.uk

http://dx.doi.org/10.15277/bjd.2016.108 cialist and primary care clinicians and allow rapid access to support and advice. Patient and health professional education also enables care to be delivered effectively, locally to the patient. Future challenges include managing the evergrowing demands within a shrinking financial envelope and developing prevention measures to reduce the prevalence of diabetes in the area.

$\mathrm{Br} J$ Diabetes 2016;16:188-192

Key words: Diabetes National Service Framework, quality improvement, quality outcomes framework, diabetes education, National Diabetes Inpatient Audit

\section{Introduction}

In the UK there are around 4 million people with diabetes, predicted to rise to over 5 million in the next decade. ${ }^{1}$ Diabetes complications are multisystem, placing an increasing burden on cardiovascular, renal, ophthalmological, podiatric and vascular services. Rapid growth in the prevalence of type 2 diabetes (T2D) in the UK places a significant challenge on diabetes services. At a time of significant financial constraints, the only way by which services have been able to meet the challenge of rapid growth in the prevalence of diabetes is to innovate.

Multifactorial intervention and structured personal care can reduce complications in patients with T2D. ${ }^{2}$ The challenge of translating evidence from clinical trials into real improvements in diabetes care in the face of a growing prevalence of the disease is one faced by all services, particularly those serving deprived inner city areas. In this article we describe a journey of improvement in diabetes care in Tower Hamlets.

\section{Tower Hamlets}

Tower Hamlets is an inner London borough serving a population of approximately 260,000 , of whom over $30 \%$ are south Asian and $10 \%$ are African Caribbean. ${ }^{3}$ The borough is the third most deprived in the UK. The population is relatively young due to high birth rates, and significant numbers of children live in families dependent on benefits ( $45.7 \%$ in Tower Hamlets compared with $8.4 \%$ in Richmond). In terms of wider determinants of health, much of the housing in the borough is council housing (59\%), and $33 \%$ of private rented accommodation is classified as 'non-decent'. The rate of crime is $37 \%$ higher than the average for London, and $23 \%$ of the working age population have no qualifications (com- 
pared with $12 \%$ in London).

Concerning health behaviours, $27 \%$ of the population smoke (21\% nationally), although the proportion is higher among Bangladeshi males (40\%). Tower Hamlets has the highest death rate attributable to smoking in London and a standardised mortality ratio for cardiovascular death of $145.90 \%$ of the local population eat $<5$ portions of fruit or vegetable each day $(70 \%$ nationally), and only $18 \%$ participate in sport/active recreation (21\% nationally), with lowest levels in Bangladeshi females. 40\% of the white population are classified as problem drinkers compared with 20\% nationally. Four in 10 of the residents of Tower Hamlets adopt only one healthy behaviour (mainly alcohol abstinence).

By virtue of its high proportion of at-risk ethnic populations and significant social deprivation, there is a rapidly growing prevalence of T2D. In 2004 there were 4,040 people with known T2D on diabetes registers in the borough. By 2016 we now have 15,612 people with T2D and around 1,000 people with type 1 diabetes (T1D). At the present rate of growth there are likely to be around 25,000 people living with T2D in the borough by 2030.4

\section{Diabetes services in Tower Hamlets in 2004}

In 2004 it was recognised that diabetes care in the borough was suboptimal. A predominantly secondary care-based service was swamped by increasing numbers of people with diabetes being referred for routine diabetes care and follow-up. By late 2004, waiting time for a new patient appointment was approaching one year, with over 25 new referrals per week. Two diabetes nurse specialist posts were both vacant and unable to be filled. There was no formal educational programme for patients or health professionals, and poor community links. Outcomes were poor, with high rates of diabetes-related complications, and poor glucose and risk factor management. $^{5}$

\section{Drivers for change - the managed clinical network}

It was clear that services had to change, and the publication of the Diabetes National Service Framework (NSF), ${ }^{6}$ along with additional resources from the Quality and Outcome Framework (QOF), led to the development of a Managed Clinical Network (Tower Hamlets Diabetes NSF Implementation Board) with representatives from primary and secondary care, the Primary Care Trust, public health and people with diabetes. This board met monthly, with the remit of implementing the Diabetes NSF priorities locally. These included $100 \%$ coverage of retinopathy screening, every practice to have a register of diabetes patients to provide call and recall, structured clinical care and a personal diabetes record and care plan. The NSF identified that key steps to achieving this were to develop a local diabetes network involving all stake holders including patients, undertake a local baseline assessment of services, participate in comparative and local audit, develop a workforce skills profile and develop training and education programmes for patients and health professionals.

Following a number of patient participation groups, it became clear that people with diabetes were unhappy with local services. They identified two main areas for improvement: (1) they wished to be looked after near to their home (by their GP or nurse), but to see a specialist rapidly if they had problems; and (2) they wished to have access to information, support and education in their own language.

The Tower Hamlets Diabetes NSF Implementation Board developed a business case for additional investment into diabetes care, with the aim of increasing support to primary care to provide routine structured diabetes care for the majority of patients with T2D, and to have clear referral pathways for specific groups of patients who should have access to more specialist diabetes care. In addition, specialist support for primary care was deemed necessary, particularly for the transition period when patients were being handed back to primary care for ongoing management. Additional investment was made at a time when resources were available for transformational change in services, aiming to reduce referrals to secondary care. It is unlikely that such change could have occurred without additional resources. Importantly, however, it was recognised that diabetes services were poorly resourced in the area prior to investment.

\section{Model of care}

The model of diabetes care for Tower Hamlets is outlined in Figure 1. A number of components to this model were developed:

1 Clear pathways for referral: We made clear which patients needed to be referred for specialist diabetes care, including T1D, pumps and transitioning patients; renal disease (chronic kidney disease stage $3 b$ or lower with problematic diabetes control), active foot disease, pregnancy or pre-pregnancy counselling and inpatients with diabetes.

2 GP networks: All practices in Tower Hamlets were divided into locality-based networks (or clusters) comprising 4-5 practices. These networks had some additional resources to deliver 'Network Enhanced Services' (NES). The Diabetes Care Package

Figure 1. Model of care for diabetes in Tower Hamlets

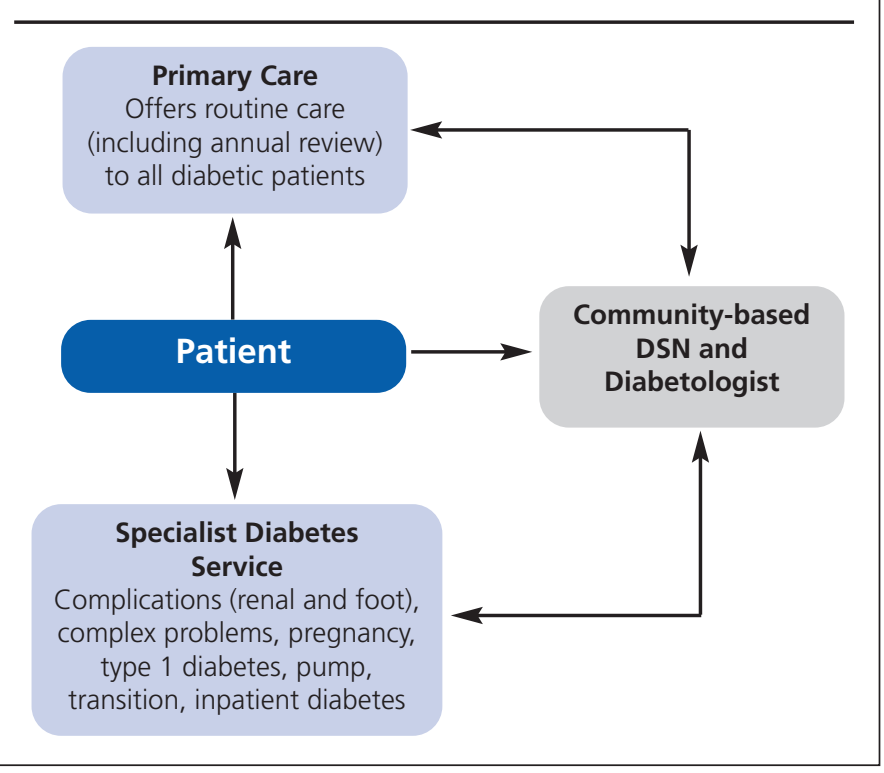


is one such NES. Networks received some additional funds for delivering care planning according to the Year of Care model, of which Tower Hamlets was a pilot site. ${ }^{7}$ They receive additional payments according to attaining key performance indicators (KPIs) in blood pressure and cholesterol (aiming to achieve 50\% of all patients with total cholesterol $\leq 4.0 \mathrm{mmol} / \mathrm{L}$ and blood pressure $\leq 140 / 80 \mathrm{mmHg}$ ), glycated haemoglobin (network average $\mathrm{HbA} 1 \mathrm{c} \leq 58 \mathrm{mmol} / \mathrm{mol}$ ), retinal screening (aiming for $>80 \%$ ) and care planning (aiming for $>90 \%$ of patients having a care plan in the last 15 months).

3 Educational support for primary care: All health professionals delivering diabetes care in the area were expected to have attended the Warwick Certificate in Diabetes Care or equivalent. The diabetes specialist team offers further educational support for health professionals, such as training in care planning, insulin commencement, protected learning time educational events, etc.

4 Educational support for patients: The specialist team offer structured diabetes education for patients in Bengali and English for T1D and T2D.

5 Specialist support for primary care: One consultant programmed activity per week was set aside for community-based support and additional community-based diabetes specialist nurse (DSN) provision was developed.

a. Network multidisciplinary team meetings (MDTs): a consultant in diabetes attends each network six times per year to undertake MDTs with GPs, practice nurses, dietitians, DSNs and diabetes psychologists. These two-hour meetings offer an opportunity for review of the KPIs for the diabetes care package, an update on clinical issues, guidelines, treatments, etc and the opportunity for primary care clinicians to discuss difficult clinical cases.

b. Community-based DSN clinics: poor glucose control, insulin or other injectable treatment commencement, problem solving clinics.

c. Email advice: consultant offers rapid email advice for all health professionals in the area dealing with diabetes. All emails are answered within 24 hours.

d. Rapid access to telephone advice in hours via the diabetes nurse team or consultant and out of hours via the endocrinology and diabetes registrar on call.

e. Virtual clinics: review of practices poor glucose control patients to improve management of challenging patients.

f. Community-based MDT clinics: occasionally a practice will request the consultant to undertake a combined consultation with a GP or practice nurse for patients about whom they are particularly concerned (e.g. frequent non-attenders or house-bound patients).

6 IT infrastructure

a. The Queen Mary Clinical Effectiveness Group produces monthly data of GP performance against the Diabetes Care Package KPIs, thus allowing networks and practices to be aware of areas that require further work (http://www. blizard.qmul.ac.uk/research-groups/253-clinical-effectiveness-group.html). These data are reviewed in the twomonthly network MDT meetings. b. Recently the diabetes specialist team has moved to using EMIS Web as their diabetes clinical information system. All general practices in the area are using EMIS Web and, as a result, we have a genuine shared record which allows seamless diabetes care, review of non-attenders' clinical records and easy access to advice for primary care clinicians.

\section{Outcomes}

Outcomes of this work have been previously published with respect to primary care, ${ }^{8}$ and some results are repeated here (Figures 2-5). In 2007/8 the QOF scores for diabetes were among the lowest in the country. There was low uptake in diabetic retinal screening, high rates of diabetes-related hospital admissions and little evidence of systematic diabetes care along the lines suggested by the Diabetes NSF. Data are available over the period from 2008/9 to 2012/3, during which time the network-delivered diabetes care packages were embedded. During this time the proportion of patients with a care plan completed in the previous 15 months rose from 10\% to $88 \%$. The proportion of patients attending for digital retinal screening in the previous 15 months rose from $72 \%$ to $82.8 \%$. The average $\mathrm{HbA}_{1 \mathrm{c}}$ value of all T2D patients in Tower Hamlets fell from 62 $\mathrm{mmol} / \mathrm{mol}(7.80 \%)$ to $60 \mathrm{mmol} / \mathrm{mol}(7.66 \%)$, which did not meet the care package target of $58 \mathrm{mmol} / \mathrm{mol}(7.5 \%)$. The combined target for cholesterol $\leq 4.0 \mathrm{mmol} / \mathrm{l}$ and blood pressure $\leq 140 / 80 \mathrm{mmHg}$, however, rose from $35.3 \%$ to $46.1 \%$. Figures 3 and 4 illustrate the improved performance of Tower Hamlets before and after the start of the intervention compared with regional and national figures. In 2012 Tower Hamlets ranked top among all Primary Care Trusts in England for cholesterol and blood pressure control. Of note is that retinal screening improvement has been less pronounced than that of other performance indicators. The reason for this is unclear, but is similar to that seen in many retinal screening programmes nationwide.

Other positive outcomes include the reduction in referrals to specialist care from 25 per week in 2004 to around six per week cur-

Figure 2. Percentage of people with diabetes with annual retinopathy screening completed. Tower Hamlets rates compared with London and England (QOF data, no exception reporting)

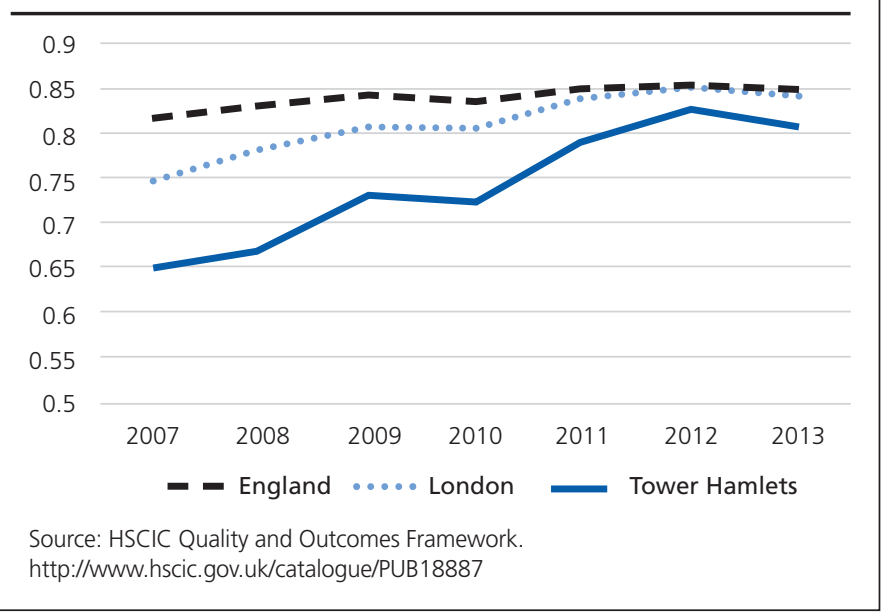


Figure 3. Proportion of people with diabetes with cholesterol $\leq 5 \mathrm{mmol} / \mathrm{L}$. Tower Hamlets compared with London and England (QOF data, no exception reporting)

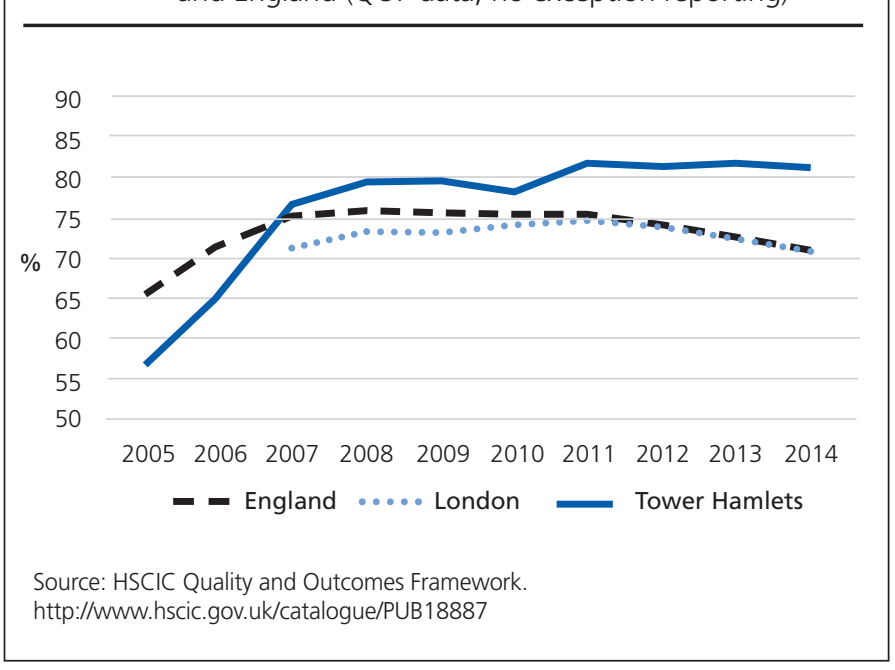

Figure 4. Proportion of people with diabetes with blood pressure $\leq 145 / 85 \mathrm{mmHg}$. Tower Hamlets compared with London and England (for 2012 QOF target $\leq 150 / 90 \mathrm{mmHg}$ ) (QOF data, no exception reporting)

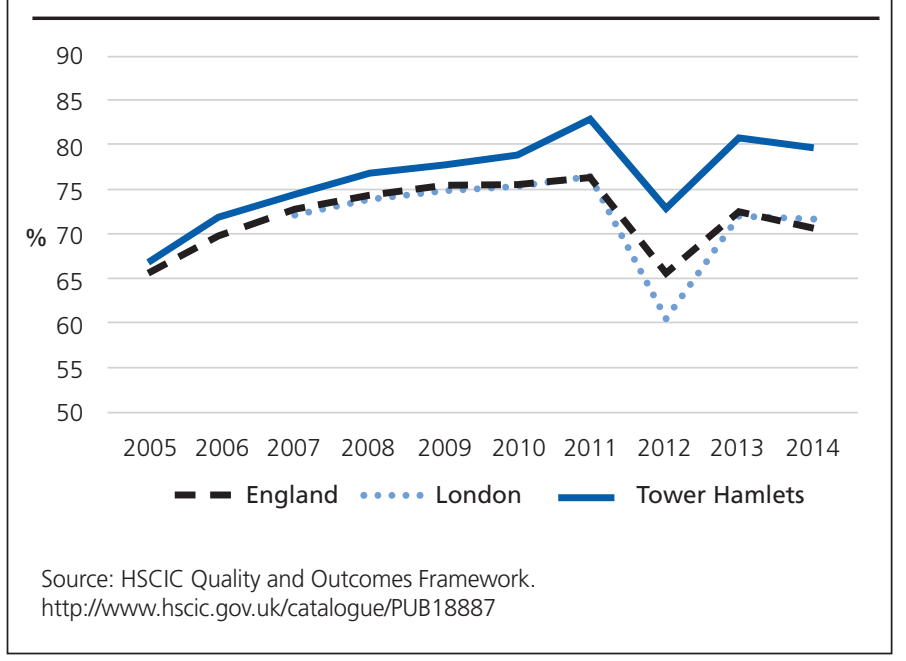

rently, and a routine waiting time of around four weeks (urgent referrals are generally seen on the same day), despite the rapid rise in diabetes prevalence. Satisfaction surveys from patients undertaken yearly by the networks show high levels of satisfaction with care planning (which has been sustained at over $90 \%$ in recent figures), diabetes education and specialist diabetes services.

\section{Inpatient diabetes care}

Whilst community-based diabetes care in Tower Hamlets has improved over the last decade due to a partnership approach between patients, clinicians and other stakeholders, the same cannot be said until recently for inpatient diabetes services. National Inpatient Diabetes Audit (NaDIA) surveys have shown poorer outcomes and lower satisfaction levels with diabetes care when patients with dia-
Figure 5. Proportion of people with diabetes with $\mathrm{HBA} 1 \mathrm{c} \leq 9 \%$ ( $72 \mathrm{mmol} / \mathrm{mol}$ ). Tower Hamlets compared with London and England (QOF data, no exception reporting)

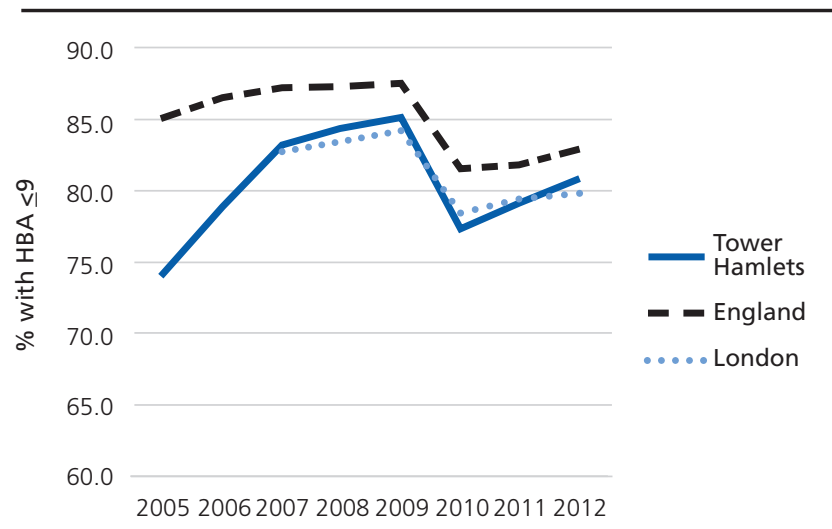

Data from QOF (http://www.gpcontract.co.uk)

DCCT laboratory harmonization occurred in 2009, with adjustment of values

betes are admitted to hospital. In particular, there were higher than average insulin and medication errors and poor staff knowledge of diabetes.

Using the Commissioning for Quality and Innovations (CQUIN) framework from 2013 to 2015 for extra resource, a multifaceted improvement plan was designed. An inpatient diabetes care plan was used, along with weekly consultant-led MDT/ward rounds, an updated designated diabetes drug chart and all inpatient trust policies were aligned to national policies. Outcomes were audited in 2015 following two years of implementation (Table 1) and showed a significant improvement in most key performance indicators, many of which exceeded the national average.

Therefore, a multifaceted approach to inpatient diabetes care along with focused extra staffing resources has led to a significant improvement in inpatient diabetes outcomes in Tower Hamlets, and the majority of parameters now significantly exceed national figures.

\section{Lessons learnt and next steps}

A decade ago, in the face of a "tsunami" of diabetes, our service was failing its patients and local clinicians who were exasperated by a poor secondary care service. Redesign of the service was necessary to enable upskilling of primary care professionals and to allow specialists to undertake specialist roles. The fundamental shift of the centre of gravity of diabetes care from a hospital-based service to primary care-based services has been attempted in many areas with varying degrees of success. ${ }^{9}$ Our philosophy has been to try and improve services based on patients' wishes for a more holistic community-centred approach, underpinned by patient and professional education and specialist support for primary care clinicians. We believe the approach of ensuring rapid access to specialist advice (to a consultant or community-based DSN) has been fundamental to the success of our approach and has enabled trust to be built between patients, primary and specialist care. We have also aimed to develop a 'diabetes service without walls' where patients are at the centre 
Table 1 Comparison of key performance indicators in National Inpatient Diabetes Audit between 2013 and 2015 at the Royal London Hospital (RLH)

\begin{tabular}{lllll}
\hline & $\mathbf{2 0 1 3}$ in RLH & $\mathbf{2 0 1 3}$ National average & $\mathbf{2 0 1 5}$ in RLH & 2015 National average \\
Seen by diabetes team & $27.8 \%$ & $34.7 \%$ & $57 \%$ & $35.5 \%$ \\
Foot risk assessment & $18.2 \%$ & $42.4 \%$ & $57 \%$ & $33 \%$ \\
Oral medication prescription errors & $47.7 \%$ & $12.4 \%$ & $4.8 \%$ & $15.7 \%$ \\
Oral medication management errors & $35.5 \%$ & $12.9 \%$ & $20 \%$ & $13.5 \%$ \\
Insulin prescription errors & $33 \%$ & $26.9 \%$ & $7.5 \%$ & $30.9 \%$ \\
Insulin management errors & $28.4 \%$ & $27.8 \%$ & $19.5 \%$ & $16.2 \%$ \\
Mild hypoglycaemia & $22.1 \%$ & $20 \%$ & $12.2 \%$ & $20 \%$ \\
Severe hypoglycaemia & $8.5 \%$ & $9.3 \%$ & $4.1 \%$ & $9.8 \%$
\end{tabular}

of their care and can obtain advice and education rapidly at times of need. Rather than investing resources in more specialists in diabetes care, we have adopted the approach of building partnerships with local primary health professionals to enhance their skills in managing people with diabetes and offer them support when needed. The diabetes MDT has been fundamental to this model. The MDT enables relationships between specialists and primary care to be developed, and has enabled a significant reduction in referrals to specialist care in spite of a rapid rise in the prevalence of diabetes in the area. Close working relationships with primary care have also enabled our service to highlight areas where the service has needed to develop, such as inpatient diabetes, and bid for scarce additional resources in order to improve poorer services.

Whilst we have seen an improvement in diabetes services and outcomes in the borough, major clinical challenges remain. In the face of the rapid growth in diabetes prevalence, primary care and specialist services are being put under increasing pressure to manage patients with more scarce resources. Newer methods of care, such as web-based consultations used elsewhere in East London, are being considered. ${ }^{10}$ We recognise that services for complex patients such as those with significant renal problems (dialysis and renal transplantation) are lacking structure and coherence due to patients attending from many areas of East London. At the other end of the spectrum, services for pre-conception counselling and prevention of diabetes in those at high risk are areas that need urgent attention. We recognise that the latter has been a particularly neglected area since the transfer of public health services from the health sector to the local council. The greatest challenge that faces us in our local area - and, indeed, nationally - is to help develop services that will enable us to reverse the trend in the incidence of diabetes, particularly in high-risk groups.

Acknowledgements We are grateful to Dr John Robson and Dr Sally Hull of the Queen Mary University of London Clinical Effectiveness Group for supplying us with the QOF data. We are indebted to the multidisciplinary team of GPs, nurses, healthcare assistants, podiatrists, dietitians, psychologists and administrative staff whose dedicated work underpins the improvements in care for patients in the area.

Conflict of interest None. Funding None.

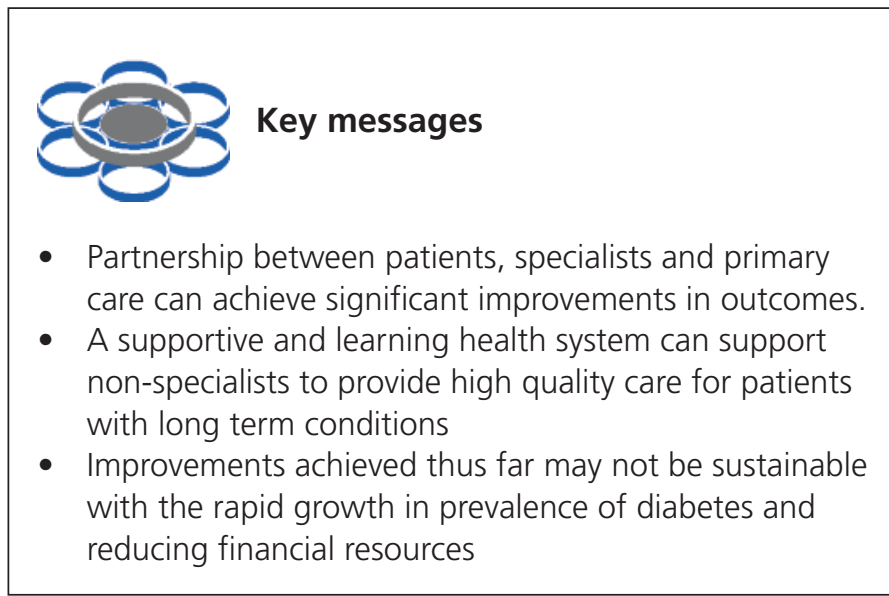

\section{References}

1. Diabetes UK. Facts and figures (Dec 2015). https://www.diabetes.org.uk/ About us/What-we-say/Statistics/ (accessed 7 Jan 2016).

2. Gaede $\mathrm{P}$, Lund-Andersen $\mathrm{H}$, Parving HH, Pedersen O. Effect of a multifactorial intervention on mortality in type 2 diabetes. $N$ Engl J Med 2008;358:580-91. http://dx.doi.org/10.1056/NEJMoa0706245.

3. Tower Hamlets Borough Profile 2015. http://www.towerhamlets.gov.uk/ Ignl/community_and_living/borough_profile/borough_profile.aspx (accessed 7 Jan 2016).

4. London Health Observatory. Cited January 2013. www.Iho.org.uk (accessed 7 Jan 2016).

5. Chowdhury TA, Lasker SS, Mahfuz R. Ethnic differences in control of cardiovascular risk factors in patients with type 2 diabetes attending an Inner London diabetes clinic. Postgrad Med J 2006;82:211-15. https://doi.org/10.1136/pgmj.2005.036673

6. Department of Health. National Service Framework for Diabetes. https://www.gov.uk/government/uploads/system/uploads/attachment_da ta/file/198836/National_Service_Framework_for_Diabetes.pdf (accessed 7 Jan 2016).

7. Hunn A. Diabetes Year of Care Evaluation Report. Tribal Consulting, 2011

8. Hull S, Chowdhury TA, Mathur R, Robson J. Improving outcomes for patients with type 2 diabetes using general practice networks: a quality improvement project in east London. BMJ Qual Saf 2014;23:171-6. http://dx.doi.org/10.1136/bmjqs-2013-002008

9. Gelding SV, Vijayaraghavan S, Davison C, Chowdhury TA. Community diabetes: an East London perspective. J R Soc Med 2005;98:96-100. https://doi.org/10.1258/jrsm.98.3.96

10. Vijayaraghavan $S, O$ 'Shea T, Campbell-Richards D, et al. DAWN: Diabetes Appointments via Webcam in Newham. Br J Diabetes Vasc Dis 2015 15:123-6. http://dx.doi.org/10.15277/bjdvd.2015.026 